\title{
Multi-Radio Multi-Channel Protocol for Emergency Wireless Mesh Network
}

\author{
Feng Jin ${ }^{1}$ \\ Beijing General Research Institute of Mining and Metallurgy, Beijing 102600, China \\ University of Science and Technology Beijing, Beijing 100083, China \\ Beijing Key Laboratory of Nonferrous Intelligent Mining Technology, Beijing 102600, China \\ E-mail: jinfeng26370090163.com

\section{Xiao Lv} \\ Beijing General Research Institute of Mining and Metallurgy, Beijing 102600, China \\ Beijing Key Laboratory of Nonferrous Intelligent Mining Technology, Beijing 102600, China \\ E-mail: gracepenguine126.com
}

\section{Xu Liu}

Beijing General Research Institute of Mining and Metallurgy, Beijing 102600, China

Beijing Key Laboratory of Nonferrous Intelligent Mining Technology, Beijing 102600, China

E-mail: I iuxu12240bgrimm.com

\begin{abstract}
In order to solve the limited energy and low throughput problems of the battery-powered wireless mesh network (WMN), an IEEE 802.11n based multi-radio multi-channel energy balancing and high throughput protocol (EBHT) for battery-powered emergency WMN is presented in this paper. EBHT optimizes the route path planning to achieve the energy balance by taking WMN nodes' residual energy, client accessed condition and route paths' link quality into account. It also assigns different high quality channels for adjacent nodes to improve the client communication performance and introduces dual-radio multi-channel allocation algorithm to increase network throughput. The EBHT's performance is evaluated by designing and implementing an IEEE 802.11n based WMN node. The results show that EBHT can improve WMN's throughput, enhance the communication reliability and optimize the node energy consumption. Compared with the traditional Mesh protocols, EBHT is more suitable for battery-powered emergency WMN.
\end{abstract}

CENet2017

22-23 July 2017

Shanghai, China

\footnotetext{
${ }^{1}$ Speaker

${ }^{2}$ This research was supported by International S\&T Cooperation Program of China (Grant 2015DFA60330).
} 


\section{Introduction}

Wireless Mesh Network (WMN) is a hot research topic in both academic and industry field. It's a self-organization, self-matching wireless network which is easily to be deployed. WMN consists of mesh clients and mesh routers, while the mesh routers form the wireless backbone and cooperate with the wired network to provide the wireless coverage for all mesh clients. WMN has been prevalently applied in industry monitoring, emergency services, smart city, and etc ${ }^{[1]}$.

In most cases of emergency WMN, multi-hop wireless connection is widely used due to its extension of the communication distance. However, with the increasing quantity of wireless hops, the WMN's throughput will decrease accordingly. The throughput problem is primarily caused by the half-duplex and channel contention in the single radio nodes. Single radio nodes can't receive and transmit packets at the same time[2-4]. They must receive first and then transmit in order to finish a reply. Similarly, single channel wireless networks usually have low transmit rate due to the neighboring interference.

And, due to the higher demand for high-speed transmission, IEEE 802.11n standard has been adopted to deploy the emergency WMN. IEEE 802.11n has several special features of improvements for high throughput such as multiple input multiple output (MIMO), channel bonding and MAC layer frame aggragation. It can provide up to $600 \mathrm{Mbps}$ PHY transfer rate when using 4 data streams and $40 \mathrm{MHz}$ channel[5]. Whereas, IEEE $802.11 \mathrm{n}$ consumes more power than IEEE $802.11 \mathrm{a} / \mathrm{b} / \mathrm{g}$ does. The power consumption is an important element for emergency WMN nodes, especially for the battery powered router nodes. If the battery runs out of power, the routers will stop working, and even the neighboring routers and clients' communications will be influenced too. Furthermore, IEEE $802.11 \mathrm{n}$ is more suitable for pointto-point communication than multi-hop communication, therefore, the multi-hop IEEE 802.11n comes to be a key point of WMN research.

In recent researches, many routing layer and MAC layer protocols were proposed to enhance the performance of IEEE 802.11n Mesh networks. Among these, IEEE has been working on IEEE 802.11s standard to improve the multi-hopping capacity. The IEEE 802.11s task group (TG) aims to standardize the WMN by defining the PHY and MAC layer protocols to support information transmissions under self-configureured mesh network topology[1,6]. Figure.1 shows the mesh network topology where devices are connected with many redundant interconnections between network nodes. But, the newest standard has not been widely applied and spread.

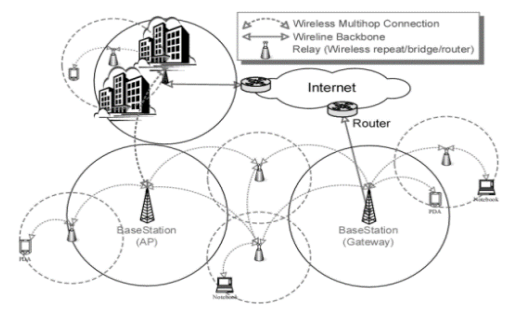

Figure.1:Mesh Network Topology

AN Le proposed a load-aware routing metric (LARM) for multi-radio wireless mesh networks. LARM can help find paths that are better in terms of balance load and reduction of the flow interference by capturing the differences in the transmission rates, packet loss ratio, intra / inter flow interference and traffic loading in the multi-radio WMN[7]. A. P. Subramanian formulated and addressed the channel assignment problem in multi-radio multi-channel WMN, 

and designed the centralized and distributed algorithms which assign channels to communication links in the network with the objective of minimizing network interference ${ }^{[8]}$. C. E. Perkins presented an on-demand distance vector routing protocol (AODV) which has already been adopted as Ad hoc networks RFc standard. The AODV avoids the counting-to-infinity problem of other distance-vector protocols by using sequence numbers on route updates.

With multiple radios, nodes can receive and transmit packets simultaneously. Some multiradio WMN protocols equip the wireless nodes with more than one radio or wireless interface, and assign the radios with centralized, static, and dynamic channel schemes to improve WMN throughput and the capacity of resisting interference[3,10]. Nevertheless, these protocols hasn't considered the extra power consumption caused by the multiple radios.

Some multi-channel MAC layer protocols were proposed to improve the performance of the wireless networks[11-13]. These protocols can make good use of the channel resource, but fail to consider the real-time quality of the channel. If nodes switch to the channels which are in bad conditions (interference, over-loaded, etc.), the network's communication performance will be further decreased.

In this paper, we proposed an EBHT cross-layer protocol for battery-powered emergency WMN which can not only increase the mesh network's throughput by adopting multiple channels assignment algorithm and equipping the router nodes with multiple IEEE 802.11n radios, but also improve the energy efficiency and prolong the battery-powered WMN router nodes' lifetime by using high reliable low-power multi-hop routing paths selection algorithm. In addition, we have developed an IEEE 802.11n-based WMN testbed to evaluate the EBHT's performance.

\section{Characteristics of the Emergency WMN}

The Emergency WMNs are usually deployed in harsh areas or accident scenes to provide temporary emergency communications. The characteristics of this application are described as follows:

1) Due to lack of external power supplies in above-mentioned areas, most emergency WMN devices are powered by the self-contained batteries.

2) Multi-hop network is needed due to the network's irregular and varying topology.

3) High throughput is desired to transmit and relay the real-time videos, voices, etc.

4) The network's transmission must have high reliability and quality to guarantee the emergency QoS (quality of service).

\section{EBHT Cross-Layer Protocol}

In most WMN applications, multi-radio protocols can significantly improve the network's transmission performance. In theory, the simultaneous using of multiply radios over different channels can provide a liner throughput increasing to the most extent. But in the real world application, the more radios nodes are used, the higher energy consumption, hardware cost and data process will be caused. For most battery-powered emergency WMN router nodes, it is unsuitable to greedily pursue the high performance by equipping the nodes with large number of radios.

EBHT introduces a dual-radio assignment mechanism to reach the balance between the performance requirement and power consumption. With two IEEE $802.11 \mathrm{n}$ radios, router nodes 

can receive and transmit simultaneously, and full-duplex communication can be achieved. To make the best usage of the radios, EBHT makes following requirements:

1) $R_{A}^{I}$ (Radio A of router node $\mathrm{n}_{i}$ ) works at $5-5.8 \mathrm{GHz}$ to form the backbone of the emergency WMN. The network is deployed in the harsh areas where the external wireless interference is very little. Therefore, we take no account of external interference during forming the backbone.

2) $R_{B}^{I}$ (Radio B of router node $\mathrm{n}_{i}$ ) works at $2.412-2.483 \mathrm{GHz}$ to provide the mesh clients with wireless coverage.

3) All nodes' radios have the same receiving and transmitting capabilities.

Moreover, to meet the high data throughput, transmission reliability and energy efficiency requirements of the emergency WMN, EBHT combines the reliable-efficient multiply hop)routing path selection algorithm (REMH ) with the dual-radio based multi-channel MAC protocol (DRMC). REMH takes link quality of transfer route, energy-efficiency and CPU loading into account during route selection to build more stable and reliable backbone networks. DRMC allots each neighbor WMN node best quality and non-overlapping channels to improve the utilization and increase networks' throughput and reliability.

\subsection{REMH Routing Path Selection Algorithm}

REMH keeps on dynamically building the network between each active WMN node and the gateway node $\mathrm{GW}$, and only the radios that work at 5-5.8 Ghz are involved in this process. Each WMN node sustains a neighbor node information table which is shown in Table 1.

\begin{tabular}{|c|l|}
\hline Fields & \multicolumn{1}{|c|}{ Describes } \\
\hline IP address & Neighbor node's IP address \\
\hline MAC address & MAC address of neighbor node's 5Ghz radio \\
\hline Channel & Channel used by neighbor node's 2.4Ghz radio \\
\hline Accumulation packet received rate & Accumulation packet received rate between neighbor node and GW \\
\hline energy-performance coefficient & Performance coefficient of route from neighbor node to GW \\
\hline remaining energy rate & Neighbor node's current remaining energy percentage \\
\hline Accessed client number & Current accessed client number of neighbor node \\
\hline
\end{tabular}

Table 1:Nerghbor Node Information

In order to strengthen the WMN's communication reliability, REMH uses packet received rate to evaluate the wireless link quality. In this evaluation system, link quality $Q$ and packet received rate $P_{i, j}\left(\mathrm{CH}_{\text {index }}\right)$ are defined as follows:

$$
\begin{gathered}
Q_{i}=\prod_{k=i}^{G W} P_{k}=P_{i, j} \cdot Q_{j} . \\
P_{i, j}\left(C H_{\text {index }}\right)=\frac{h_{i, j}}{\mu_{i} \times T} .
\end{gathered}
$$

Where $\mathrm{n}_{j}$ is the forwarding node of $\mathrm{n}_{i}, Q_{i}$ is the product of packet received rate of every forwarding nodes between $\mathrm{n}_{i}$ and GW, $P_{i, j}\left(\mathrm{CH}_{\text {index }}\right)$ refers to the rate of received packet $h_{i, j}$ from $\mathrm{n}_{i}$ to $\mathrm{n}_{j}$ within the time $\mathrm{T}$ on channel $C H_{\text {index }}$, while $\mu_{i}$ presents the packet sending speed of $\mathrm{n}_{i}$. 
To improve the energy efficiency, $\mathrm{n}_{i}$ will detect its residual power percentage $E_{\mathrm{i}}$ in real time and use it as an important factor to transmit the route selection. Considering in real-world application each node with different energy consumption speed, this paper assumes that CPU running consumption of each node is nearly the same.Thus the radio's transmitting and receiving data comes to be the major operation of energy consumption. The energy consumed by radio receiving and transmitting data can be calculated as follows[14]:

$$
\begin{aligned}
& E_{T x}(l, d)=E_{\text {radio }}(l)+E_{T x-a m p}(l, d) . \\
& E_{R x}(l)=E_{\text {radio }}(l)=l E_{\text {elec }} . \\
& E_{T x-\text { amp }}(l, d)=\begin{array}{l}
l \varepsilon_{f s} d^{2}, d<d_{0} \\
l \varepsilon_{m p} d^{4}, d>d_{0}
\end{array}
\end{aligned}
$$

Where $E_{T x}(l, d)$ and $E_{R x}(l)$ denote the node's energy consumption of transmission and receiving $l$ bit data over distance $d, E_{T x}(l, d)$ is composed of wireless radio circuit's energy consumption $E_{\text {elex }}$ and power amplification circuit's energy consumption $E_{T x-a m p}(l, d)$ of sending $l$-bit data. If $d$ is smaller than threshold $d_{0}, E_{T x-a m p}(l, d)$ adopts the free space propagation model, otherwise, $E_{T x-a m p}(l, d)$ adopts the multipath attenuation propagation model.

According to (3.3), $\mathrm{n}_{i}$ 's energy consumption $R_{\mathrm{i}}$ is directly proportional to $l$, that is to say, $R_{\mathrm{i}}$ will grow with the increasing of $\mathrm{n}_{i}$ 's accessed client number $N_{i}$. And the CPU loading will also get increased which may cause bigger transmission delay when the WMN node connects to more clients. So, during the process of selecting the routing paths, REMH uses $N$ as an important index to evaluate the energy consumption and CPU's load with

$$
\zeta_{j, i}=Q_{i} \cdot E_{j} \cdot \frac{1}{\left(N_{j}+1\right)^{\alpha+1}}=P_{i, j} \cdot Q_{j} \cdot E_{j} \cdot \frac{1}{\left(N_{j}+1\right)^{\alpha+1}} .
$$

Where $\alpha$ denotes the CPU's load-delay parameter which can be adjusted according to CPU's processing ability and the WMN's real-time requirement. $\zeta_{j, i}$ is $\mathrm{n}_{j}$ 's energyperformance coefficient of becoming to be $\mathrm{n}_{i}$ 's forwarding node. The bigger $\zeta_{j, i}$ is, the longer survival time and better overall performance $\mathrm{n}_{i}$ 's routing path through $\mathrm{n}_{j}$ will have.

The following steps give the details of REMH.

Step 1: GW periodically broadcasts a set of network test messages NT_MSG which consist of node information and time stamp.

Step 2: Each WMN node receives and forwards GW's NT_MSG, then calculates the packet receive rate based on NT_MSG.

Step 3: GW sends out the network configureuration message NC_MSG after finishing the network testing. Once receiving the NC_MSG, WMN node sends out the route matching message RM_MSG which consists of source node ID, source node residual power, source node accessed client number, source node accumulation packet received rate and wireless coverage channel to its neighbor node immediately.

Step 4: On receiving $\mathrm{n}_{j}$ 's first NC_MSG, $\mathrm{n}_{i}$ will calculate $\zeta_{j, i}$ by (3.6) and select the node that has the largest $\zeta$ to be its forwarding node. Then, $n_{i}$ will update its neighbor node 

information table, and send the routing require message RR_MSG to the selected node and NC_MSG to its neighbor nodes.

Besides, the newly joined node will broadcast network access request message, and select the just node with the largest $\zeta$ to be its forwarding node based on the NC_MSG responded by the neighbor nodes during current communication period. Within this communication period, the newly joined node will not affect other nodes' communication. When some nodes are out of the network during the period, the nodes that forward data to these dropped nodes before will re-select the forwarding node as the newly joined nodes.

\subsection{DRMC MAC Protocol}

DRMC mainly works during the WMN nodes' wireless coverage period. Each node dynamically maintains an available channel queue when the channel elements are ordered upon the local channel quality and neighbor nodes' channel usage condition. The local channel quality is evaluated as follows:

$$
E_{t}\left(C H_{\text {index }}\right)=\beta E_{t-1}\left(C H_{\text {index }}\right)+(1-\beta) P_{\mathrm{t}}\left(\mathrm{CH}_{\text {index }}\right), \quad \beta \quad(0,1) .
$$

Where $P_{\mathrm{t}}\left(\mathrm{CH}_{\text {index }}\right)$ and $E_{t}\left(\mathrm{CH}_{\text {index }}\right)$ denote the packet received rate of the node in channel $\mathrm{CH}_{\text {index }}$ within the time slot and the channel $\mathrm{CH}_{\text {index }}$ 's quality estimation. $\beta$ is the historical channel quality impact factor. The influence of the historical channel quality on the current quality estimation can be controlled by adjusting $\beta$. The larger the $E_{t}\left(\mathrm{CH}_{\text {index }}\right)$ is, the better the channel $\mathrm{CH}_{\text {index }}$ performance will be.

After forwarding the routing path selection is finished, each node gets its neighbor nodes' ID. During the coverage channel competition, each node identifies the sequence of choosing channel by comparing its node and neighboring nodes' ID. The larger the node's ID is, the higher priority the node has. And the node will choose the channel queue's first channel to be the coverage channel. Once finishing the channel selection, node will broadcast a channelselected message CS_MSG immediately to inform its neighbor nodes of the channel selection result. Then, all nodes that have received CS_MSG will remove the neighbor node's coverage channel and the adjacent channels to the end of its local channel queue.

When the network topology changes, or the channel quality gets worse, node will reassign the coverage channel by DRMC to make full use of the high quality channel and try to avoid using the interferential channel for coverage of neighbor nodes.

\section{Performance Evaluation}

An IEEE $802.11 \mathrm{n}$ based Mesh node testbed was designed to evaluate the EBHT protocol's performance. This testbed is equipped with AR9350 and AR9592, which are both 2.4/5 GHz dual-band 2×2 IEEE 802.11n RFs. Besides, AR9350 is integrated with a $533 \mathrm{MHz}$ processor which is used to process the data and manage the communication protocol. Furthermore, to the faciliate the computer connection, an ethernet controller QCA8327 is mount to AR9350 through the RGM II interface. The hardware testbad is shown in Figure.2, and its structure is shown in Figure 3. 


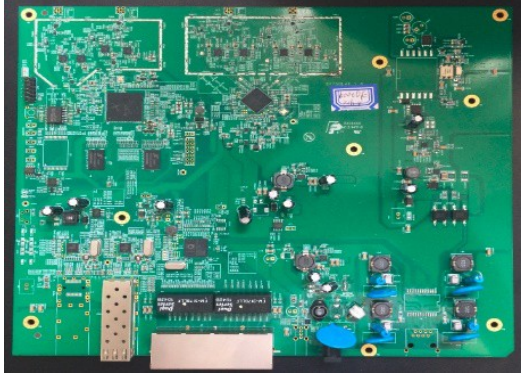

Figure 2: Testbed Hardware

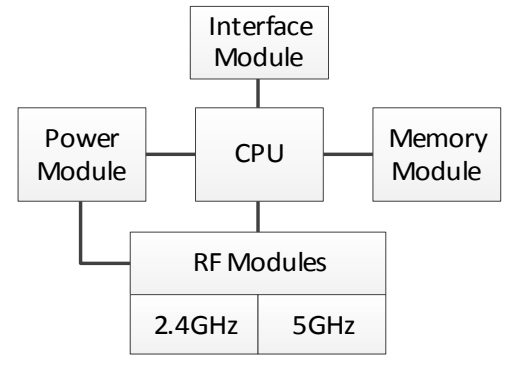

Figure 3: Testbed Hardware Structure

16 Mesh node testbeds and 4 interference sources were used to build the test Mesh network. Every Mesh node is equipped with a $12000 \mathrm{mAH}$ Lithium-ion battery and a $11 \mathrm{dBi}$ omni antenna. For each node, AR9350 worked at $2.4 \mathrm{GHz}$ frequency band to realize wireless access, while AR9592 worked at $5 \mathrm{GHz}$ frequency band to form the backbone of the WMN. The client was simulated by the computer with the NETGEAR WNA3100 Wi-Fi card, and the performance parameters were recorded by the NETIQ CHARIOT.

All nodes were equipped with full charge state batteries and deployed on the open-sided site to test the communication energy consumption and survival time of WMN nodes. Figure 4 shows the standard deviation of nodes' residual energy percentage at different periods of time under AODV and EBHT. Difference value of residual energy percentage among EBHT nodes was smaller than that of AODV nodes at every time period. Compared with AODV protocol, EBHT achieved a better energy-balanced performance. Figure. 5 shows the survival node number in different time period under AODV and EBHT. EBHT nodes got longer lifetime due to the EBHT's energy-balanced character which can postpone the GW nearby nodes' death time by collecting the client accessed number and residual energy percentage during route planning. Moveover, it helps the WMN improve the communication stability and efficiency.

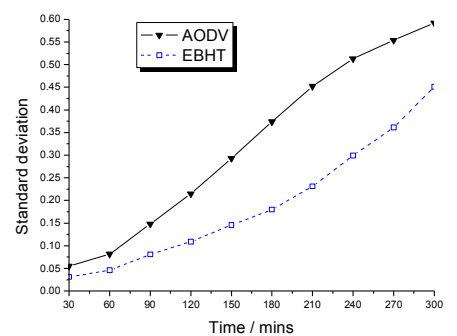

Figure 4:Standard Deviation of Energy Percentage

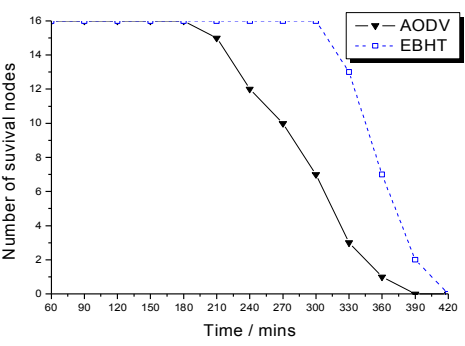

Figure 5: Survival Node Number

Figure 6 and Figure 7 present the network's average packet drop rate (PDR) of different number of UDP clients and during different periods of time respectively. When the number of the whole network's accessed client is small (less than 25), PDR of EBHT and AODV are nearly the same. When the number of accessed clients increases, it's apparent that EBHT gets lower PDR than AODV due to DRMC multi-channel allocate strategy. DRMC makes good use of the channel resource and reduces the internal co-channel conflict and the external wireless interference. Furthermore, PDR is an important factor for REMH to plan forwarding path. Therefore, EBHT can significantly improve the reliability of the WMN's communication. 

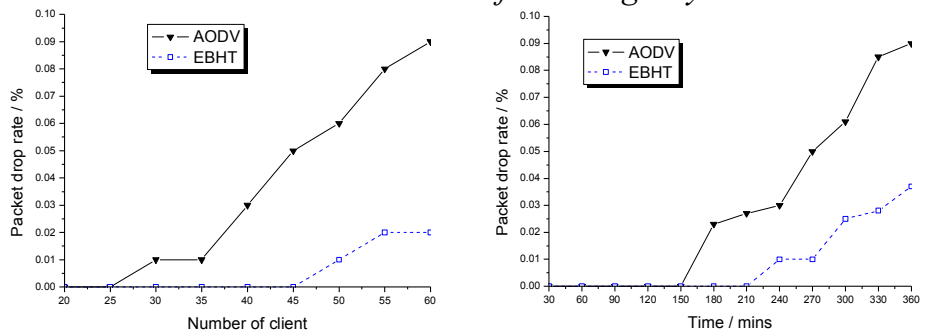

Figure 6:PDR under Different Number of Client
Figure 7: PDR under Different

Time Period

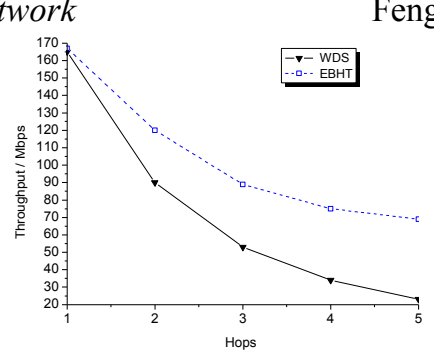

Figure 8: Network

Throughput

Figure 8 shows the throughput comparison of single-RF Wireless Distribution System (WDS) and dual-RF EBHT under the chain backbone topology. Both the EBHT and WDS throughput drop when the node hops increases, which is mainly caused by radio collision. But EBHT throughput maintains at least $30 \mathrm{Mbps}$ higher than WDS throughput from 2 hops to 6 hops with the use of dual-radio and multi-channel. EBHT's dual-radio technology can help reduce the radio collision and avoid the extra energy consumption, while EBHT's multi-channel technology and route planning algorithm can balance the channel loading and reduce the retransmitting caused by packet loss. All in all, EBHT gets good performance in improving the throughput.

\section{Conclusion}

In this paper, we proposed an IEEE $802.11 \mathrm{n}$ based multi-radio multi-channel protocol EBHT for the battery-powered emergency WMN. The EBHT fuses the reliable and efficient route path selection algorithm REMH and dual-radio based multi-channel MAC protocol DRMC to meet the lifetime and throughput needs of battery-powered WMN systems. REMH adopts nodes' energy-performance coefficiency as an important route path selection criterion to prolong WMN lifetime and improve network's packet received rate, while DRMC avoids wireless interference and improves network's throughput by assigning a non-adjacent channel to its neighboring nodes. Besides, we designed and implemented the IEEE 802.11n based dualradio WMN testbed to evaluate the EBHT's performance. The evaluation results show EBHT is suitable for battery-powered emergency WMN with its comprehensive efficient performance. It can improve WMN's throughput, enhance communication reliability and optimize node energy consumption.

\section{References}

[1] E. Hossain, K. Leung. Wireless Mesh Networks Architectures and Protocols. Springer Science\&Business Media, 2008.

[2] I. W. H. Ho, P. P. Lam, P. H. J. Chong, and S. C. Liu. Harnessing the High Bandwidth of Multiradio Multichannel 802.11n Mesh Networks. IEEE Trans on Mobile Computing, 2014,13(2): 448-456.

[3] R. Draves, J. Padhye, and B. Zill. Routing in Multi-radio, Multi-hop Wireless Mesh Networks. ACM Mobicom, 2004: 114-128.

[4] I. W. H. Ho, P. P. Lam, P. H. J. Chong, and S. C. Liu. Real-life experiments of Multi-Radio MultiChannel Wireless Mesh Networks: 802.11n is not any better than 802.11a!. Proc. of the $8^{\text {th }}$ International Conference on Information, communications and Signal Processing, 2011: 1-5.

[5] W. S. Kim, S. H. Chung. Design and Implementation of IEEE 802.11n in Multi-hop over Wireless Mesh Networks with Mulit-channel Multi-interface. Proc. of IEEE $14^{\text {th }}$ International Conference on 
High Performance Computing and Communication \& IEEE $9^{\text {th }}$ International Conference on Embedded Software and System, 2012: 707-713.

[6] "802.11s-IEEE Standard for Information Technology--Telecommunications and information exchange between systems--Local and metropolitan area networks--Specific requirements Part 11: Wireless LAN Medium Access Control (MAC) and Physical Layer (PHY) specifications Amendment 10: Mesh Networking”, IEEE Standard, 2011.

[7] A. N. Le, D. W. Kum, Y. Z. Cho, and I. S. Lee. LARM: A Load-Aware Routing Metric for multiradio Wireless Mesh Networks. Proc. of International Conference on Advance Technologies for Communications, 2008: 166-169.

[8] A. P. Subramanian, H. Gupta, S. R. Das, and J. Cao. Minimum Interference Channel Assignment in Multiradio Wireless Mesh Networks. IEEE Trans. on Mobile Computing, 2008, 7(12): 1459-1573.

[9] C. E. Perkins, E. M. Royer. Ad hoc On-Demand Distance Vector Routing. Proc. of the 2nd IEEE Workshop on Mobile Computing Systems and Applications, 1999: 90-100.

[10] M. Song, J. Wang, K. Xing, and E. K. Park. Interference-aware Broadcasting in Multi-radio Multichannel Mesh Networks. IEEE Trans on Wireless Communication. 2008, 7(12): 5473-5481.

[11] S. Wang, M. S. Wang, H. S. Hong, and Y. Ma. Environmental Monitoring System Based on Sensor Networks Using Multi-channel MAC Protocol. Proc. of International Conference on Wireless Communications, Networking and Mobile Computing. 2009: 1-4.

[12] D. Gong, M. Zhao, and Y. Yang. A Multi-channel Cooperative MIMO MAC Protocol for Wireless Sensor Networks. Proc. of IEEE $7^{\text {th }}$ International Conference on Mobile Adhoc and Sensor Systems, 2010: 11-20.

[13] J. Alapati, S. N. Merchant, and U. B. Desai. Multi Channel MAC Protocol for Broadcast transmissions in Vehicular Networks. Proc. of IEEE International Conference on Vehicular Electronics and Safety, 2012: 421-426.

[14] W. R. Heinzelman, A. Chandrakasan, and H. Balakrishnan. Energy-efficient Communication Protocol for Wireless Microsensor Networks. Proc. of the Hawaii International Conference on System Sciences, 2000: 1-10. 